\title{
ON LINE INTEGRALS AND DIFFERENTIAL EQUATIONS, ESPECIALLY THOSE OF DYNAMICS
}

BY D. C. LEWIS, JR.

1. Introduction and Formulation of the Problem. Recently a formula was given, exhibiting a Cartan relatively invariant line integral for a non-holonomic conservative dynamical system.* I wish to show that this formula is a special case of a more general formula which will here be developed. The formula itself is not explicitly that of a relatively invariant line integral, even in the special case previously treated. Nevertheless, under certain restrictions it can undoubtedly be put into that form. $\dagger$

Let us consider the system of differential equations

$$
\frac{d x_{1}}{X_{1}}=\frac{d x_{2}}{X_{2}}=\cdots=\frac{d x_{m}}{X_{m}},
$$

together with an arbitrary linear differential form $\sum_{i=1}^{m} A_{i} d x_{i}$, where the $X_{i}\left(x_{1}, \cdots, x_{m}\right)$ and $A_{i}\left(x_{1}, \cdots, x_{m}\right)$ are of class $C^{\prime \prime}$ in a region $R$. Let $\Gamma_{0}$ be a closed curve $\ddagger$ in $R$, whose parametric equations are given, say, by

$x_{i}=x_{i}{ }^{(0)}(\tau), \quad 0 \leqq \tau \leqq 1, \quad x_{i}{ }^{(0)}(0)=x_{i}{ }^{(0)}(1), \quad(i=1, \cdots, m)$.

Consider the tube $T$ of trajectories of (1) which pass through $\Gamma_{0}$. Let $\Gamma_{u_{1}}$ be any other similar closed curve embracing this tube; that is, $\Gamma_{u_{1}}$ cuts each trajectory of the tube the same number of times as $\Gamma_{0}$ and in the same order. $\S$ The whole of $T$ between $\Gamma_{0}$ and $\Gamma_{u_{1}}$ inclusive is assumed to be contained in $R$.

* A. E. Taylor, On the integral invariants of non-holonomic dynamical systems, this Bulletin, vol. 40 (1934), pp. 735-742.

$\dagger$ For a complete treatment and bibliography of the related subject of integral invariants see E. J. Cartan's book, Legons sur les Invariants Intégraux, 1922.

$\ddagger$ The curve $\Gamma_{0}$ may have double points. For definiteness it may be assumed that the $x_{i}^{(0)}(\tau)$ are of class $C^{\prime \prime}$ except for a finite number of corners.

$\S$ It is to be noticed that $T$ is a singular tube (in a certain obvious sense) whenever $\Gamma_{0}$ cuts a trajectory more than once. This is necessarily the case when $m=2$. 
In its most general form the formula to which we allude gives a relation between the values of the line integral

$$
\int \sum_{i=1}^{m} A_{i} d x_{i}
$$

taken over the path $\Gamma_{0}$ and its value over the path $\Gamma_{u_{1}}$, the two paths being taken in proper senses.

2. Derivation of the General Result. $T$ may be represented parametrically by equations of the form

$$
x_{i}=x_{i}(\tau, u), \quad x_{i}(0, u) \equiv x_{i}(1, u),
$$

where the lines $\tau=$ constant represent the trajectories, the line $u=0$ represents the curve $\Gamma_{0}$, and the line $u=u_{1}$ (where $u_{1}$ is any preassigned number other than zero) represents the curve $\Gamma_{u_{1}}$, as anticipated in the notation.* Along a trajectory on $T$ we must have the following relations deduced from (1):

(3) $\frac{\partial x_{i}}{\partial u}=U(\tau, u) X_{i}\left[x_{1}(\tau, u), \cdots, x_{m}(\tau, u)\right],(i=1,2, \cdots, m)$.

The factor of proportionality $U$ is at most a function of $\tau$ and $u$. For constant $u$ equations (2) represent a closed curve $\Gamma_{u}$. Let

$$
J(u)=\int_{\Gamma_{u}} \sum_{i=1}^{m} A_{i} d x_{i}=\int_{0}^{1}\left(\sum_{i=1}^{m} A_{i} \frac{\partial x_{i}}{\partial \tau}\right) d \tau .
$$

We find by a straightforward process of differentiation, integration by parts, and substitution from (3), that

$$
\frac{d J}{d u}=J^{\prime}(u)=\int_{0}^{1}\left(\sum_{i=1}^{m} U \beta_{i} \frac{\partial x_{i}}{\partial \tau}\right) d \tau,
$$

where

$$
\beta_{i}=\sum_{j=1}^{m}\left(\frac{\partial A_{i}}{\partial x_{j}}-\frac{\partial A_{j}}{\partial x_{i}}\right) X_{j} .
$$

Thus we get the required relation connecting $J\left(u_{1}\right)$ and $J(0)$ :

* This can obviously be done in an infinite number of ways, such that $x_{i}(\tau, u)$ is of class $C^{\prime \prime}$ except on a finite number of trajectories corresponding to the corners of $\Gamma_{0}$, where $\partial x_{i} / \partial u$ is continuous but $\partial x_{i} / \partial \tau$ and the second partial derivatives may not be. 


$$
J\left(u_{1}\right)=J(0)+\int_{0}^{1} d \tau \int_{0}^{u_{1}}\left(\sum_{i=1}^{m} U \beta_{i} \frac{\partial x_{i}}{\partial \tau}\right) d u .
$$

In case $R$ is a small neighborhood of a point where not all the $X$ 's vanish simultaneously, it is possible to write the iterated integral on the right as a line integral about $\Gamma_{u_{1}}$. This representation is rather artificial, however, and we shall not insist upon it.* Furthermore the existence of a relatively invariant line integral in this case can yield no invariantive properties of the system (1). It is hardly more than a reflection of the fact that the system (1) can be transformed in such a region into the form $d x_{1} / 0=d x_{2} / 0=\cdots=d x_{m-1} / 0=d x_{m} / 1$.

3. An Important Special Case. We now consider the special case when $X_{m} \equiv 1$. In this case it is usual to write $t$ in place of $x_{m}$. From (3) and (5), we see that

$$
\left\{\begin{aligned}
\frac{\partial t}{\partial u}= & U(\tau, u) \\
\beta_{i}= & \sum_{j=1}^{m-1}\left(\frac{\partial A_{i}}{\partial x_{j}}-\frac{\partial A_{j}}{\partial x_{i}}\right) X_{j}+\frac{\partial A_{i}}{\partial t}-\frac{\partial A_{m}}{\partial x_{i}}, \\
& (i=1,2, \cdots, m-1), \\
\beta_{m}= & \sum_{j=1}^{m-1}\left(\frac{\partial A_{m}}{\partial x_{j}}-\frac{\partial A_{j}}{\partial t}\right) X_{j} .
\end{aligned}\right.
$$

4. The Application to Dynamics. Let us apply the special case of the preceding paragraph to the equations of motion of a dynamical system (not necessarily conservative), written in the following form:

$$
\frac{d q_{i}}{d t}=\frac{\partial H}{\partial p_{i}}, \quad \frac{d p_{i}}{d t}=-\frac{\partial H}{\partial q_{i}}+Q_{i}, \quad(i=1,2, \cdots, n),
$$

taking $q_{i}=x_{2 i-1}, p_{i}=x_{2 i}, X_{2 i-1}=\partial H / \partial p_{i}, X_{2 i}=-\left(\partial H / \partial q_{i}\right)+Q_{i}$, $(2 n=m-1)$, where $H$ and the $Q$ 's are functions of the $q$ 's, $p$ 's, and $t$. The linear differential form which we consider along with (8) is $\sum_{i=1}^{n} p_{i} d q_{i}-H d t$, so that

$$
A_{2 i-1}=x_{2 i}, \quad A_{2 i}=0, \quad A_{m}=A_{2 n+1}=-H .
$$

* Taylor analogously makes the tacit assumption that the left hand side of his equation (15) is a line integral; loc. cit., p. 740. 
A straightforward calculation based on (7) shows us that

$$
\beta_{2 i-1}=Q_{i}, \quad \beta_{2 i}=0, \quad \beta_{m}=\beta_{2 n+1}=-\sum_{i=1}^{n} \frac{\partial H}{\partial p_{i}} Q_{i} .
$$

Hence, if

$$
J(u)=\int_{\Gamma_{u}} \sum_{i=1}^{n} p_{i} d q_{i}-H d t
$$

we see from (4) that

(9) $J^{\prime}(u)=\int_{0}^{1}\left[\sum_{i=1}^{n} Q_{i} \frac{\partial q_{i}}{\partial \tau}-\sum_{i=1}^{n} \frac{\partial H}{\partial p_{i}} Q_{i} \frac{\partial \imath}{\partial \tau}\right] \frac{\partial t}{\partial u} d \tau$,

and thus

(10) $J\left(u_{1}\right)=J(0)+\int_{0}^{1} d \tau \int_{0}^{u_{1}}\left[\sum_{i=1}^{n} Q_{i} \frac{\partial q_{i}}{\partial \tau}-\sum_{i=1}^{n} \frac{\partial H}{\partial p_{i}} Q_{i} \frac{\partial t}{\partial \tau}\right] \frac{\partial t}{\partial u} d u$.

This is a generalization of Taylor's formula for non-holonomic systems, as we shall show in $\$ 5$.

Another interesting result is obtained as a special case by supposing $\Gamma_{0}$ and $\Gamma_{u_{1}}$ to lie in planes $t=$ const. In this case one may take $t=u+t_{0}$ (where $t_{0}$ is a constant) and hence $\partial t / \partial u=1$ and $\partial t / \partial \tau=0$. The curve $\Gamma_{u}$ may be thought of as a closed curve $\Gamma_{t}^{\prime}$ in the phase space steadily moving with the time. It is to be noted that, for a fixed $t, \Gamma_{t}^{\prime}$ may be preassigned instead of $\Gamma_{t_{0}}^{\prime}$ as previously. An immediate corollary of (9) informs us that the time rate of change of the line integral

$$
\int_{\Gamma_{t}^{\prime}} \sum_{i=1}^{n} p_{i} d q_{i}
$$

is equal to the line integral

$$
\int_{\Gamma_{t}^{\prime}} \sum_{i=1}^{n} Q_{i} d q_{i}
$$

This result has the advantage of being expressible without the explicit introduction of the parameters $\tau$ and $u$. A result of this type can also be stated for the system of $\$ 3$.

5. Non-Holonomic Dynamical Systems. We next consider a conservative non-holonomic system subject to the constraints 
$\sum_{i=1}^{n} a_{\alpha i} \delta q_{i}+a_{\alpha} \delta t=0,(\alpha=1, \cdots, k<n)$, in which the $a$ 's are functions of the $q$ 's and $t$. We may write the equations of motion in the form (8), if we take

$$
Q_{i}=\sum_{\alpha=1}^{k} a_{\alpha i} \lambda_{\alpha}
$$

where the $\lambda$ 's are functions of the $p$ 's, $q$ 's, and $t$, chosen in such a manner that the equations (8) admit the following $k$ first integrals :

$$
\sum_{i=1}^{n} a_{\alpha i} \frac{\partial H}{\partial p_{i}}+a_{\alpha}=c_{\alpha}, \text { a constant, } \quad(\alpha=1, \cdots, k) .^{*}
$$

Thus, since $\partial H / \partial p_{i}=d q_{i} / d t$, a motion which initially satisfies the conditions of constraint will continue to do so. The motions of the non-holonomic system are precisely those motions of (8) for which $c_{1}=c_{2}=\cdots=c_{k}=0$. In considering the motions for which all these constants are not necessarily zero, we shall, however, be considering also certain motions which are not kinematically possible, that is, which do not satisfy the conditions of constraint. If we multiply (12) by $\lambda_{\alpha}$ and sum over $\alpha$, we obtain

$$
\sum_{i, \alpha} a_{\alpha i} \lambda_{\alpha} \frac{\partial H}{\partial p_{i}}+\sum_{\alpha} a_{\alpha} \lambda_{\alpha}=\sum_{\alpha} c_{\alpha} \lambda_{\alpha .}
$$

But this may be written with the help of (11) in the form

$$
\sum_{i=1}^{n} Q_{i} \frac{\partial H}{\partial p_{i}}=\sum_{\alpha=1}^{k}\left(c_{\alpha}-a_{\alpha}\right) \lambda_{\alpha}
$$

Substituting from (11) and (13) into (10), we find that

* The $\lambda$ 's may be calculated as follows: Differentiate (12) totally with respect to $t$ and substitute for the derivatives of the $p$ 's and $q$ 's their values as given by (8). In virtue of (11) the result is a system of $k$ linear equations in the unknowns $\lambda_{1}, \cdots, \lambda_{k}$. In the most important case where the kinetic energy can be written as a positive definite form in the velocities, the determinant of this system can be shown to be different from zero, the $k$ equations of constraint being linearly independent. The determinant can even be written in diagonal form by a preliminary normalization of the equations of constraint. See G. Prange, Die Allgemeinen Integrationsmethoden der Analytischen $\mathrm{Me}$ chanik, Encyklopädie der Mathematischen Wissenschaften, IV Mechanik, 2 (1935), p. 558. 


$$
\begin{aligned}
J\left(u_{1}\right)=J(0)+\int_{0}^{1} d \tau \int_{0}^{u_{1}}\left[\sum_{i=1}^{n}\right. & \left(\sum_{\alpha=1}^{k} a_{\alpha i} \lambda_{\alpha}\right) \frac{\partial q_{i}}{\partial \tau} \\
& \left.-\left(\sum_{\alpha=1}\left(c_{\alpha}-a_{\alpha}\right) \lambda_{\alpha}\right) \frac{\partial t}{\partial \tau}\right] \frac{\partial t}{\partial u} d u .
\end{aligned}
$$

Since $c$ 's may have different values along different trajectories of $T$, they are here to be considered as functions of $\tau$. If we take all the $c$ 's equal to zero, thus considering only the actual motions of the non-holonomic system, we have exactly the result of Taylor, save for a difference in notation.*

6. Characterization of Differential Equations by Line Integrals. Going back to the general case of $\$ \$ 1$ and 2 , we now state in conclusion that the validity of (4), or (6), for all tubes $T$ characterizes completely the differential equations (1), provided that the m-rowed determinant,

$$
\left|\frac{\partial A_{i}}{\partial x_{j}}-\frac{\partial A_{j}}{\partial x_{i}}\right|, \quad(i, j=1, \cdots, m),
$$

is not zero. For, suppose the $A$ 's and $\beta$ 's are given such that (4) holds for all tubes $T$. Then, $U\left(\partial x_{i} / \partial \tau\right)$ may be taken almost arbitrarily, and the equations (5) result. But these are sufficient to determine the $X$ 's in terms of the $A$ 's and $\beta$ 's. The details are left to the reader.

In the more restricted case of $\$ 3$, it is only necessary that the $(m-1)$-rowed determinant,

$$
\left|\frac{\partial A_{i}}{\partial x_{j}}-\frac{\partial A_{j}}{\partial x_{i}}\right|, \quad(i, j=1, \cdots, m-1),
$$

be distinct from zero. For, in this case, $X_{m}$ is given $\equiv 1$ and equations (7) are surely sufficient to determine the other $X$ 's.

This latter condition is fulfilled in the case of the dynamical equations and the action line integral of $\$ 4$.

Cornell University

* Loc. cit., equation (15). Taylor's $\lambda$ 's are the negatives of ours. He also takes the curve $\Gamma_{0}$ (though not $\Gamma_{u_{1}}$ ) in a plane $t=$ const. 